\title{
Influence of snake rolling on metal flow in hot rolling of aluminum alloy thick plate
}

\author{
Pujun $\mathrm{Hao}^{1,2,{ }^{*}}$ and Jingna Liu ${ }^{1,2}$ \\ 1 Tianjin Key Laboratory for Advanced Mechatronic System Design and Intelligent Control, School of Mechanical Engineering, \\ Tianjin University of Technology, Tianjin 300384, China \\ 2 National Demonstration Center for Experimental Mechanical and Electrical Engineering Education (Tianjin University \\ of Technology), No. 391 Bingshuixi Road, Xiqing District, Tianjing 300384, China
}

Received: 11 June 2019 / Accepted: 3 August 2020

\begin{abstract}
Most asymmetrical rolling conditions should not appear in regular rolling processes, but for obtaining large deformations inside aluminium alloy thick plates, the asymmetrical rolling process is the most effective method. Snake rolling is adopted for promoting more deformation inside the plates. For exploring the deformation inside an aluminium alloy thick plate, a finite element model for simulating the process of snake rolling is established and the key influence factors are set as initial thickness, speed ratio and offset distance. The results show that deformation inside of the plate increases obviously while the thickness of plate is less than $300 \mathrm{~mm}$ after snake rolling. The speed ratio has a positive effect on promoting deformation partly inside of the plate. On the contrary, the offset distance has a negative influence by affecting the exit thickness. A formula for calculating the exit thickness after snake rolling is proposed and validated by data from the finite element models. Thus, snake rolling is suggested to be used in the downstream pass of hot rough rolling considering that the influence of thickness and the offset distance should be controlled in a reasonable range.
\end{abstract}

Keywords: Aluminum alloy / snake rolling / speed ratio / offset distance / finite element method

\section{Introduction}

As a key material in the fields of manufacturing, aerospace, military and transportation, the manufacturing of aluminium alloy plates is a crucial research topic. The rolling process is the most widely used method to obtain aluminium alloy plates with proper gauges and outstanding mechanical properties.

The typical thickness of an aluminium alloy ingot, used as the raw material for hot rough rolling, is between 400 and $630 \mathrm{~mm}$. Through several passes of rolling, the thickness of the plate can be reduced to the desired value. However, deformation along the thickness direction of the plate is seriously inhomogeneous [1]. This phenomenon is inevitable in the regular rolling process.

Snake rolling is derived from cross shear rolling [2], which is used for obtaining thinner strips than the minimum rolling gauge by increasing the shear strain inside the strip [3]. As the deformation inside of the plate is promoted, serious bending of the plate appears that limits the application of cross shear rolling. Collins and Dewhurst [4] presented a slip-line field solution for calculating the rolling force, rolling torque and outgoing curvature in

\footnotetext{
* e-mail: haounwilling@163.com
}

the asymmetrical hot rolling process. Salimi et al. [5] established a theoretical model for researching plane strain asymmetrical rolling based on a slab method and the required external loading to the plate at entry to keep the plate horizontal can be precisely predicted. More researchers adopted the finite element method with commercial software to simulate the cross shear rolling process. Shivpuri et al. [6] established an explicit finite element model for analysing the asymmetrical rolling process with rolling speed mismatch and calculating the outgoing sheet curvature. Richelsen [7] studied asymmetrical plate rolling with different friction conditions at two rolls using a numerical method. Ji et al. [8] studied the deformation mechanism of rolling with a high-speed ratio using a rigidplastic finite element method.

Snake rolling is proposed by moving the slower roll towards the exit of the deformation zone to reduce the bending curvature of the plate. Research on snake rolling mostly focuses on microstructural, textural and mechanistic properties [9]. For researching the influence of offset distance and speed ratio on deformation and curvature, a number of finite element models have been established. Ling et al. [10] studied the bending behaviour of an AA6016 aluminium alloy plate in the snake rolling process with finite element simulations. Zhang et al. [11] established 
Table 1. Rolling parameter ranges.

\begin{tabular}{ll}
\hline Rolling parameter & Range \\
\hline Initial thickness of plate & $5-400 \mathrm{~mm}$ \\
Reduction rate & $5-17 \%$ \\
Diameter of work roll & $100-1300 \mathrm{~mm}$ \\
Speed ratio & $1-1.3$ \\
Offset distance & $0-70 \mathrm{~mm}$ \\
\hline
\end{tabular}

coupled thermo-mechanical finite element models for analysing the distribution in the thickness direction of equivalent and shear strain of an aluminium alloy plate during snake rolling. Yang et al. [12] studied the deformation behaviour in snake rolling of an AA7050 aluminium alloy with finite element models using a hyperbolic sine-type constitutive law. It is found that snake rolling has a positive effect on increasing the deformation inside of the plate and decreasing the curvature. For accurately predicting the curvature and improving the feasibility of snake rolling, mathematical models have been developed. Aboutorabi et al. [13] developed an analytical approach based on a slab method considering the horizontal displacement of the roll and presented a formula to predict the outgoing sheet curvature. Wang et al. [14] established an analytical model to predict the rolling force and roll torque.

Although the influence of offset distance and speed ratio on the deformation of a plate has been studied by several methods and is well known, each of the earlier models has limitations in rolling parameters. According to former studies, the rolling parameter ranges are listed in Table 1.

It can be observed that ranges of initial thickness of the plate and the diameter of the work roll are quite large. In the practical rolling process of a thick plate, deformation occurs just beneath the upper and lower surfaces and the metal located at the centre of the plate is barely affected by the rolling force. It seems that asymmetrical rolling is necessary to promote the deformation of metal located at the centre of the plate but considering the slip and diameter limitation of the work roll, the speed ratio and offset distance have upper limits, meaning that the effect of snake rolling needs to be studied carefully.

In this study, a rigid-plastic finite element model of snake rolling is established. The proper range of the initial thickness of the plate used for snake rolling is studied firstly. Then, for that range of initial thicknesses, the influence of snake rolling on the deformation of an aluminium alloy plate is studied in detail.

\section{Finite element model}

LS/DYNA is selected for modelling in this study, considering its widespread usage. A complete finite element model consists of a geometry model, material model, contact model and loads. The parameters of the geometry model are listed in Table 2.

The deformation process of the aluminium alloy can be separated in two sections, namely, the elastic and plastic ddeformation stages. Due to the large elastic modulus,
Table 2. Parameters of geometry model.

\begin{tabular}{ll}
\hline Parameter & Range \\
\hline Initial thickness of plate & $50-300 \mathrm{~mm}$ \\
Reduction rate & $10 \%$ \\
Diameter of work roll & $300,600 \mathrm{~mm}$ \\
Speed ratio & $1,1.1,1.2$ \\
Offset distance & $0,20,40,60 \mathrm{~mm}$ \\
\hline
\end{tabular}

stress increases rapidly when strain increases in the elastic stage. At the yield point, plastic deformation appears. While in the plastic stage, stress changes a little though plastic strain increasing significantly.

Based on the deformation process, the material model is simplified as an ideal elastic-plastic model. This is because the constitutive relation between stress and strain can be affected by the temperature and strain rate. The temperature of the plate during one rolling process can be kept in a narrow interval [15] under the combined action of many factors, e.g., heat transfer, plastic deformation heat and friction heat. Thus, the temperature is determined as constant values in the simulation. In the finite element model of snake rolling, the rolling time and reduction are reasonably constant, so the strain rate is also constant. With constant temperature and strain rate, the material model can be determined as a bilinear kinematic model. The elastic modulus of the plate is set to $0.8 \mathrm{GPa}$ and the yield strength is set to $60 \mathrm{MPa}$, with no hardening effect, based on the literature [16].

Considering the large elastic modulus, the elastic deformation of work rolls can be ignored. Thus, the work roll is set as a rigid roll and built as a shell. The diameter of work roll is set as $600 \mathrm{~mm}$ based on practical production lines. The element type of both the plate and work rolls is selected as SOLID164, which has eight nodes. The plate moves to the work roll with an initial velocity of $0.8 \mathrm{~m} / \mathrm{s}$ and the speed of the upper work roll is $4.8 \mathrm{rad} / \mathrm{s}$ based on the practical rolling process.

The viscous-sliding friction model [17] in the simulation is selected to calculate the friction coefficient. The friction coefficient is calculated as follows:

$$
\mu=0.44 \times\left(1+4 e^{-0.036 v}\right)(0.0185+0.000269 T)
$$

where $v$ is the rolling speed $(\mathrm{m} / \mathrm{s})$ and $T$ is the rolling temperature $\left({ }^{\circ} \mathrm{C}\right)$.

The finite element model is shown in Figure 1.

\section{Results and discussion}

\subsection{Deformation after snake rolling with different initial plate thicknesses}

In the rolling process of an aluminium alloy thick plate, the deformation distributed in the thickness direction is quite inhomogeneous and is influenced by the comprehensive action of the roll diameter and initial thickness. Figure 2 shows the distribution in the thickness direction of equivalent plastic strain after regular rolling with different 


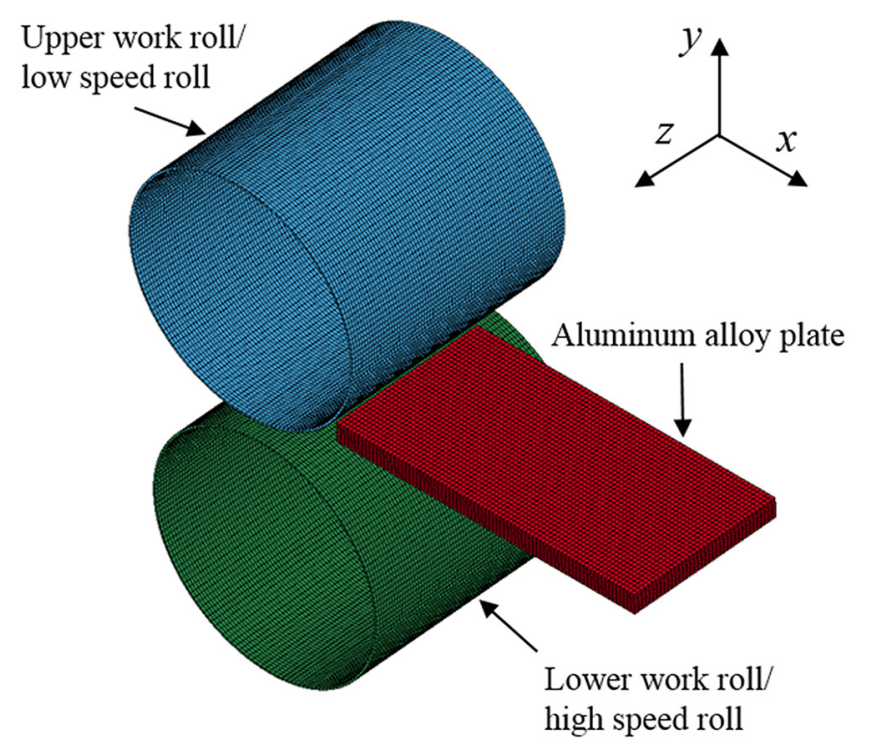

Fig. 1. Finite element model.

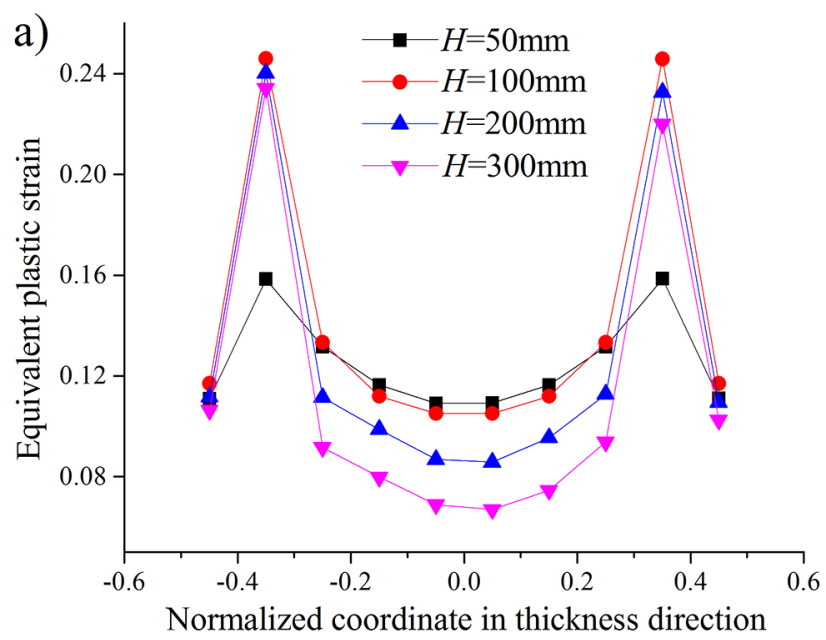

Fig. 2. Distribution of equivalent plastic strain in thickness direction after regular rolling: (a) $R=300 \mathrm{~mm}$; (b) $R=600 \mathrm{~mm}$.

roll diameters and initial thicknesses. $H$ represents the initial thickness. It can be observed that: (1) the equivalent plastic strain increases from the centre to upper and lower surfaces and reaches a maximum beneath these two surfaces; (2) with initial thickness increasing, the equivalent plastic strain at the centre of the plate decreases; (3) with roll diameter increasing, the equivalent plastic strain at the centre of the plate increases.

As stated by rolling theory, the ratio of the deformation zone contact arc length to the average thickness of the plate at the entrance and exit of the deformation zone is known as the geometric shape coefficient and is applied for predicting the distribution of deformation in the thickness direction. With an increasing geometric shape coefficient, the deformation beneath the upper and lower surfaces becomes closer to that at the centre of the plate. According to the rolling conditions shown in Figure 2, the geometric shape coefficients are listed in Table 3 .

According to the literature [18], when the geometric shape coefficient is less than 0.5 , the plastic deformation

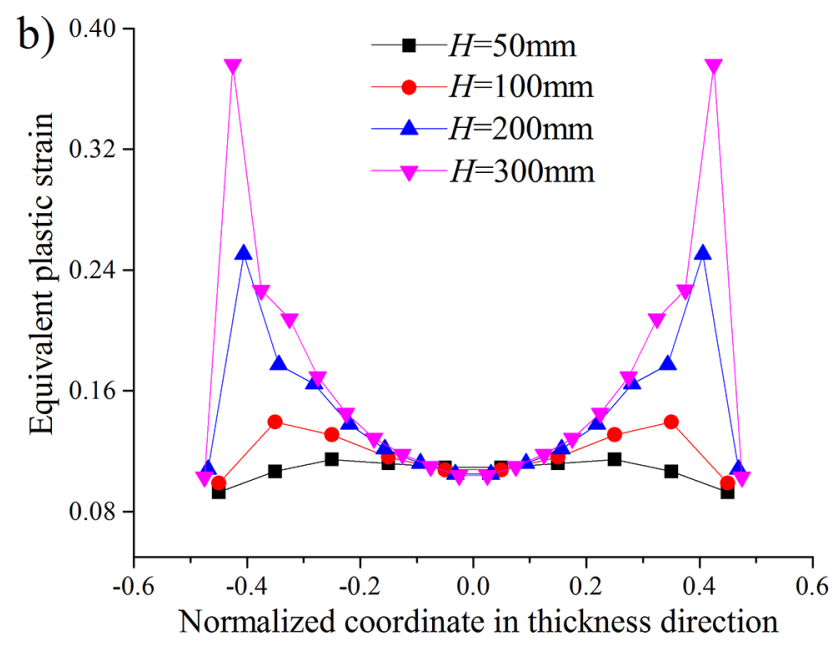

Table 3. Geometric shape coefficients with different rolling conditions.

\begin{tabular}{llll}
\hline $\begin{array}{l}\text { Initial } \\
\text { thickness } \\
(\mathrm{mm})\end{array}$ & $\begin{array}{l}\text { Roll } \\
\text { diameter } \\
(\mathrm{mm})\end{array}$ & $\begin{array}{l}\text { Reduction } \\
\text { rate }\end{array}$ & $\begin{array}{l}\text { Geometric } \\
\text { shape } \\
\text { coefficient }\end{array}$ \\
\hline \multirow{2}{*}{50} & 300 & & 1 \\
& 600 & & 1.41 \\
100 & 300 & & 0.71 \\
& 600 & \multirow{2}{*}{$10 \%$} & 1 \\
200 & 300 & & 0.5 \\
300 & 600 & & 0.71 \\
& 300 & & 0.41 \\
\hline
\end{tabular}

appears mostly beneath the upper and lower surfaces. As listed in Table 3, with increasing initial thickness or decreasing roll diameter, the geometric shape coefficient decreases. Only when both initial the thickness and roll diameter are $300 \mathrm{~mm}$ is the geometric shape coefficient less than 0.5. Thus, when the initial thickness is more than $300 \mathrm{~mm}$ and roll diameter remains unchanged, or when the initial thickness and roll diameter increase together, the geometric shape coefficient remains less than 0.5 and deformation will still appear mostly beneath the upper and lower surfaces of the plate.

Figure 3 shows the distribution in thickness direction of equivalent plastic strain after snake rolling with different initial thicknesses.

It can be obviously observed that at the side of the highspeed roll, the equivalent plastic strain increases, whereas at another side, the equivalent plastic strain decreases. The location of the minimum of equivalent plastic strain shifts towards the low-speed roll. As for deformation at the centre of the plate, the promoting effect of snake rolling on 


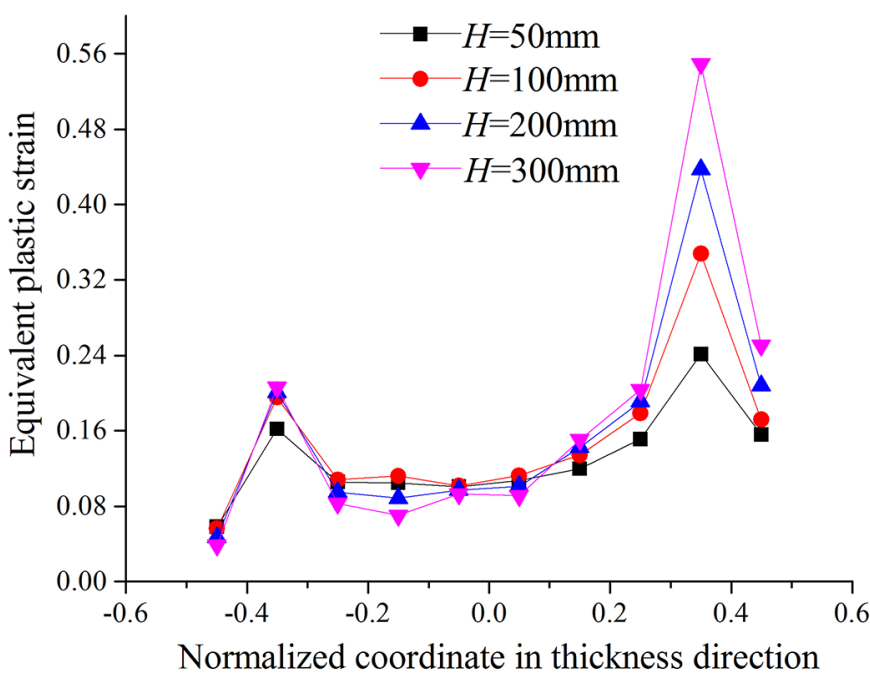

Fig. 3. Distribution in thickness direction of equivalent plastic strain after snake rolling.

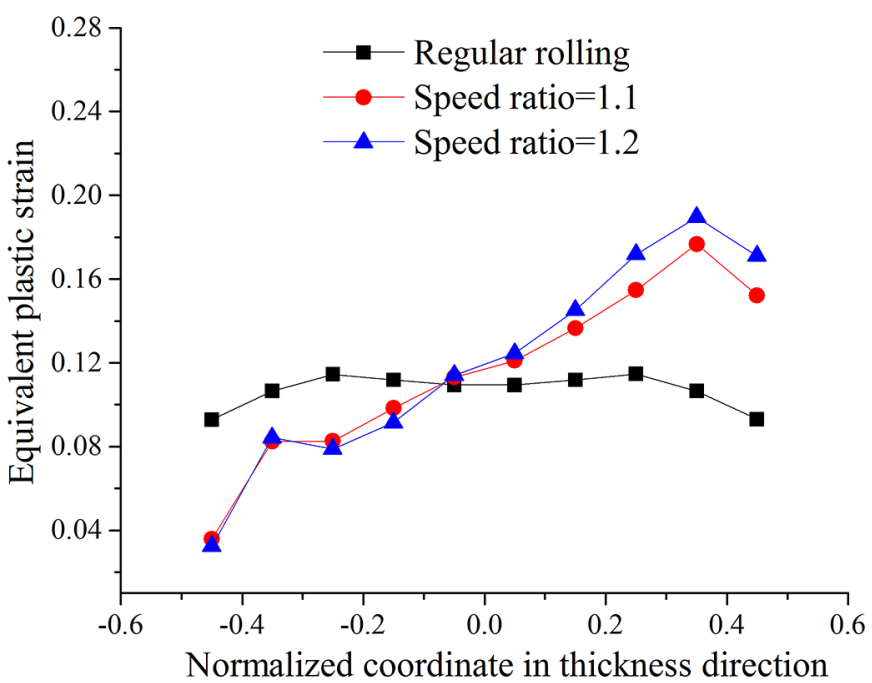

Fig. 4. Distribution of equivalent plastic strain in thickness direction after snake rolling with initial thickness and roll diameter at 50 and $300 \mathrm{~mm}$, respectively.

the equivalent plastic strain weakens with increasing initial thickness. This indicates that snake rolling can only positively promote deformation at the centre of the plate within the proper initial thickness range, instead of the whole thickness range. Therefore, in the practical rolling process, snake rolling is suggested to be adopted at the downstream pass of hot rough rolling.

\subsection{Influence of speed ratio on deformation inside the plate}

Considering the weak influence of snake rolling on thick plates, the analysis of simulation results with a small initial thickness is significant. Figure 4 shows the distributions of

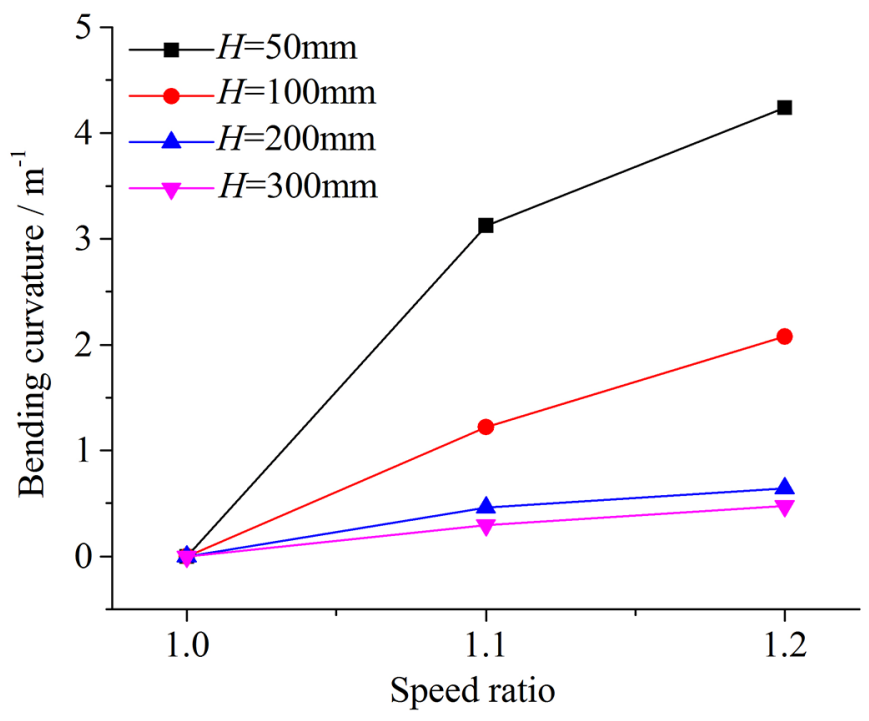

Fig. 5. Bending curvature of plate after snake rolling with offset distance and roll diameter of 0 and $300 \mathrm{~mm}$, respectively.

equivalent plastic strain under different rolling conditions when the initial thickness is $50 \mathrm{~mm}$ and the roll diameter is $300 \mathrm{~mm}$.

As shown in Figure 4, with the speed ratio increasing, the equivalent plastic strain at the side of the high-speed roll increases significantly compared to regular rolling. This indicates that more deformation appears at that side and the metal is deformed sufficiently so that better mechanical properties can be obtained. This is meaningful to aluminium alloy thick plate manufacturing. However, at the side of the low-speed roll, the equivalent plastic strain decreases. Thus, the speed ratio does not have an absolutely positive effect on improving deformation inside the plate.

In addition, with the speed ratio increasing, the equivalent plastic strain at the side of the high-speed roll increases gradually. At another side, the equivalent plastic strain decreases marginally.

Comprehensively considering the deformation in the thickness direction, after snake rolling, seriously inhomogeneous deformation will result in bending of the plate, which is typically avoided in the actual rolling process. Figure 5 shows the bending curvatures of plates being rolled with snake rolling.

It can be observed from Figure 5 that with the speed ratio increasing, the bending curvature increases obviously. This indicates that the speed ratio can result in serious bending of the plate. The promotion of deformation inside the plate caused by the speed ratio is helpful to obtain better mechanical properties and it is important to reduce the bending curvature.

In addition, with the initial thickness increasing, the increment of bending curvature decreases. This also indicates that the influence of snake rolling on deformation in the thickness direction weakens with initial thickness increasing so that the inhomogeneity of deformation in the thickness direction cannot result in serious bending. 


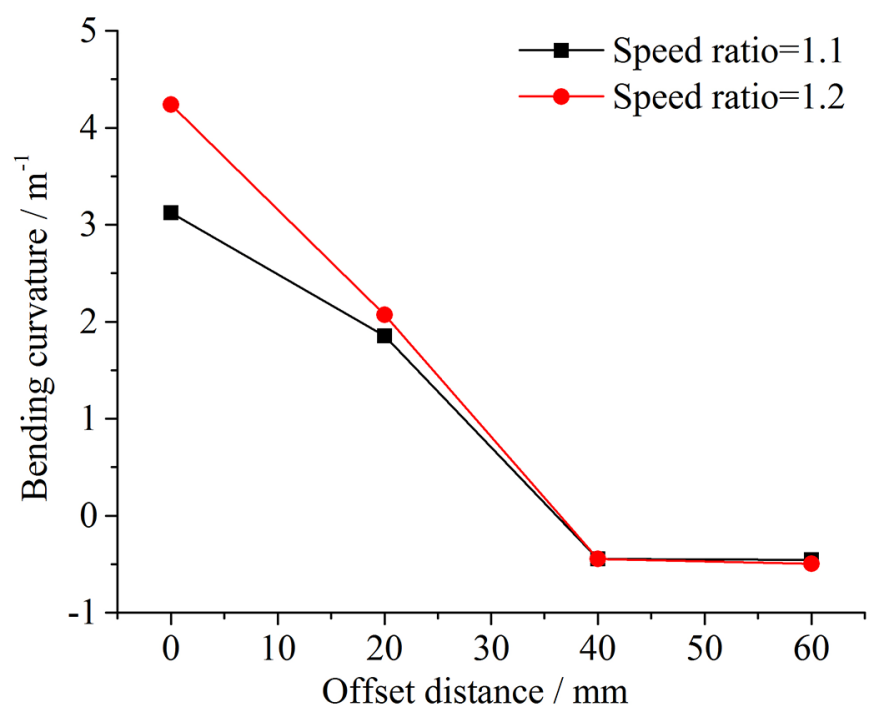

Fig. 6. Bending curvature of plate after snake rolling with initial thickness and roll diameter of 50 and $300 \mathrm{~mm}$, respectively.

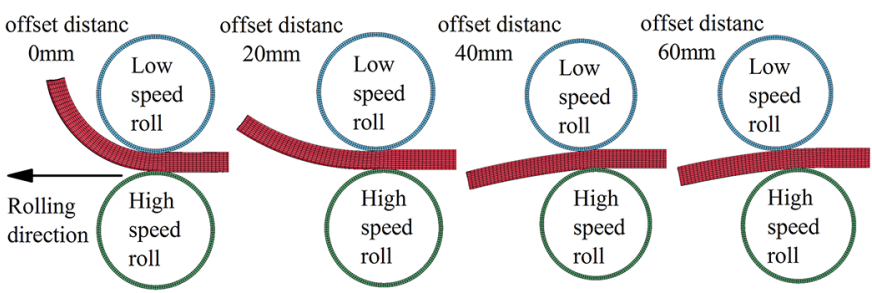

Fig. 7. Shapes of plates after snake rolling with rolling conditions shown in Figure 6.

\subsection{Influence of offset distance on deformation inside the plate}

In the snake rolling process, the offset distance is applied mainly for improving the bending caused by the speed ratio, as shown in Figure 6. It can be observed that the bending curvature of the plate after snake rolling decreases rapidly with offset distance increasing. But when offset distance reaches $40 \mathrm{~mm}$, bending curvature becomes negative which means the plate bends towards another direction. When offset distance is $60 \mathrm{~mm}$, bending curvature remain negative and the absolute value of it increases a bit.

For researching the reason that bending curvatures are almost the same when offset distances are 40 and $60 \mathrm{~mm}$, which is shown in Figure 6, the shapes of the plates and the distribution in the thickness direction of equivalent plastic strain after snake rolling with the same rolling conditions are shown, respectively, in Figures 7 and 8.

As shown in Figure 7, when the offset distance is $0 \mathrm{~mm}$, the speed of the lower roll is greater than the upper roll and the difference in speed results in the speed of the metal at the lower surface of the plate towards the rolling direction is great than that at the upper surface so that the plate bends towards the upper roll. When the offset distance is $20 \mathrm{~mm}$, the bending of the plate is improved. However, when the offset distance reaches $40 \mathrm{~mm}$, bending direction of plate is opposite. Under the current rolling condition,

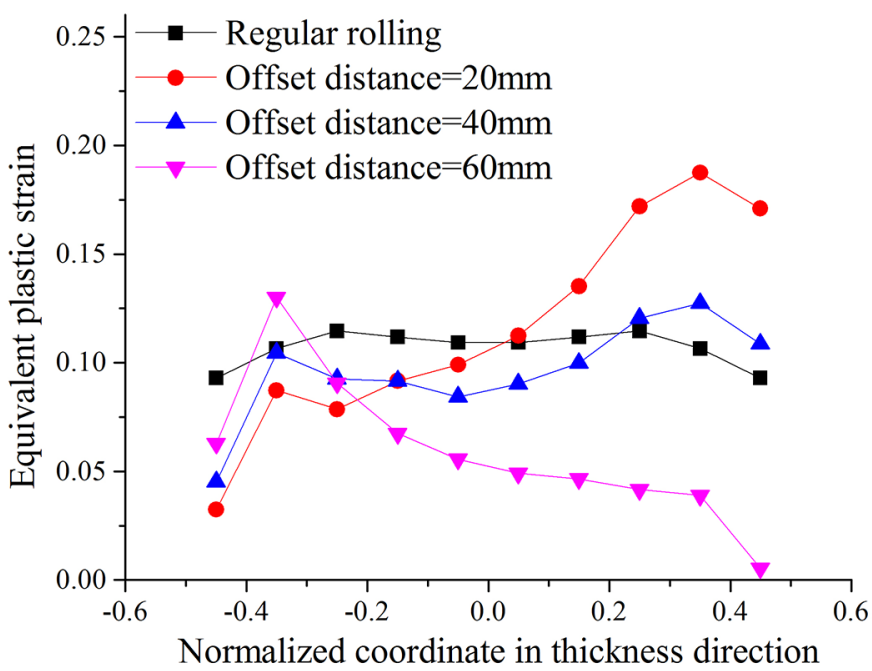

Fig. 8. Distribution in thickness direction of equivalent plastic strain with initial thickness, roll diameter and speed ratio of $50 \mathrm{~mm}, 300 \mathrm{~mm}$ and 1.1, respectively.

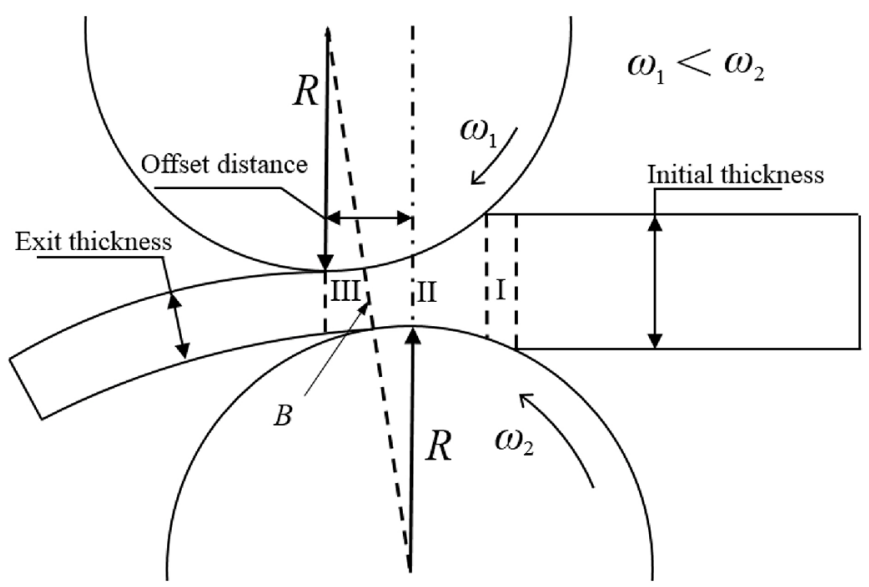

Fig. 9. Schematic diagram of deformation zone divided by contact conditions and the shortest distance between two rolls.

influence of offset distance on bending of plate is greater than speed ratio and results in switching of bending direction. In other words, compensation of offset distance for bending of the plate is beyond proper value. When the offset distance increases continuously, the bending of the plate seems to remain unchanged. However, at this time, it is notable that the exit thickness of the plate is greater than that when the offset distance is $40 \mathrm{~mm}$, observing Figure 7 carefully.

It can be observed from Figure 8 that when the offset distances are 40 and $60 \mathrm{~mm}$, equivalent plastic strain of most elements decreases. This means the total deformation of the plate decreases.

When snake rolling is adopted, the flow speed of metal located at side of high-speed roll is larger than that located at side of low speed roll so that extra shear strain resulted from difference of flow speed can affect the distribution of deformation in thickness direction. However, one of the key parameters in the snake rolling process, the offset distance, changes the shape of deformation zone, as shown in Figure 9. 


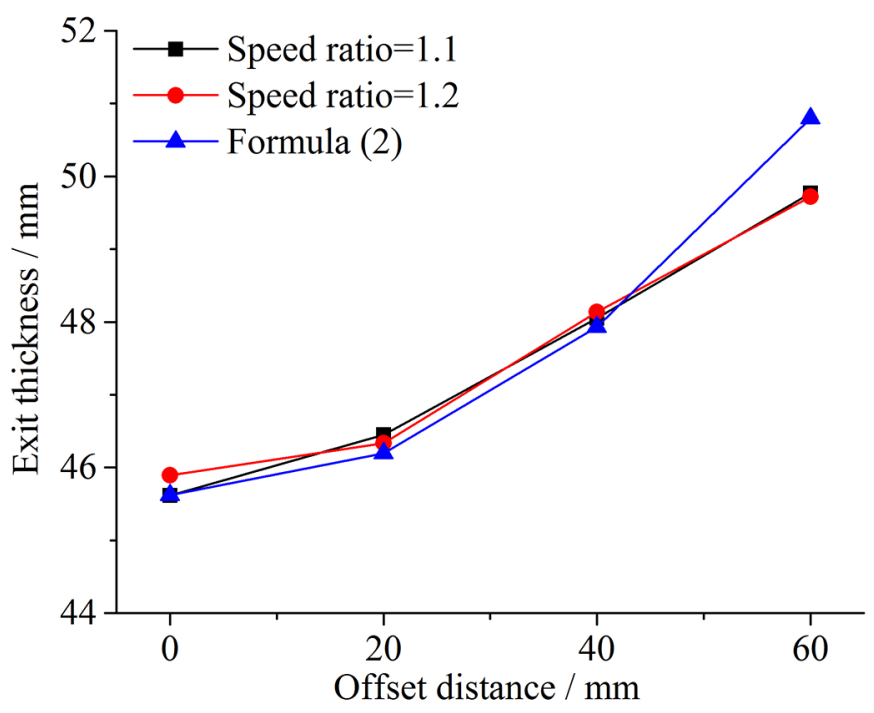

Fig. 10. Exit thickness of plate after snake rolling with initial thickness being $50 \mathrm{~mm}$.

As indicated in previous research, metal at left of zenith of lower roll is bended by work rolls, instead of being rolled. Which means thickness of plate will not reduce at left of zenith of lower roll. According to geometry of deformation zone and ignoring the influence of bending and flattening of work rolls on exit thickness, exit thickness of plate can be calculated as follows:

$$
h_{S R 2}=\sqrt{[2 R+H(1-\varepsilon)]^{2}+d^{2}}-2 R+\Delta h
$$

where $H$ is initial thickness $(\mathrm{mm}), \varepsilon$ is reduction ratio, $R$ is roll radius $(\mathrm{mm}), d$ is offset distance $(\mathrm{mm})$ and $\Delta h$ is elastic recovery after rolling $(\mathrm{mm})$. Elastic recovery can be obtained by comparison of finite element models under different rolling conditions.

It can be observed that the offset distance can lead to increasing exit thickness, which means both the true reduction ratio and deformation of plate are reduced. Although the offset distance can improve the bending of the plate after snake rolling, it has a negative effect on promoting deformation of plate according to formula (2).

By extracting displacement data of nodes from finite element models, the exit thickness after snake rolling is shown in Figure 10.

As shown in Figure 10, the exit thickness increases remarkably with offset distance increasing. The exit thickness calculated by formula (2) is closed to results of finite element models. When offset distance is $60 \mathrm{~mm}$, predicting error reaches maximum, $2.1 \%$. That is because with this offset distance, shortest distance between two work rolls is almost $50 \mathrm{~mm}$ and the plate can be hardly rolled. But this situation is not been considered when formula (2) is obtained. In available range of offset distance, the maximal error of formula (2) is $0.6 \%$ so that the precision of formula (2) can be acceptable.

\section{Conclusion}

In this study, the influence of snake rolling on deformation inside the aluminium alloy plate is studied by simulation, then the conclusions are presented as follows:

- With certain rolling conditions, deformation inside the plate decreases with initial thickness increasing. It results in that promotion of deformation inside the plate caused by snake rolling weakens with initial thickness increasing. Thus, the proper applied range of snake rolling has upper limit of initial thickness.

- Speed ratio can raise deformation at the side of the highspeed roll and reduce deformation at the side of low speed roll so that metal can be rolled sufficiently to obtain better mechanical properties. But speed ratio can lead to serious bending of plate. Then offset distance can reduce bending curvature by moving low speed roll towards roll direction. Under combined action of speed ratio and offset distance, bending of plate can be control effectively.

- Though the offset distance can effectively reduce bending curvature, it has an upper limit. Because firstly, oversize offset distance will lead to bending of plate towards opposite direction. Secondly, shape of deformation zone can be affected seriously by offset distance.

- Formula for calculating exit thickness after snake rolling is proposed and validated by data of finite element models. According to the formula, the exit thickness increases with offset distance increasing, which means the true reduction ratio reduces. This will reduce the deformation inside the plate and makes snake rolling invalid. Thus, the offset distance must be carefully chosen when snake rolling is adopted.

\section{Conflict of interest}

The authors declare that they have no conflict of interest.

This work is supported in part by National Natural Science Foundation of China (11702191), Natural Science Foundation of Tianjin (18JCQNJC75000) and China Postdoctoral Science Foundation (2018M631751)

\section{References}

[1] P. Hao, A. He, W. Sun, Formation mechanism and control methods of inhomogeneous deformation during hot rough rolling of aluminum alloy plate, Arch. Civil Mech. Eng. 18, $245-255$ (2018)

[2] J.K. Lee, D.N. Lee, Texture control and grain refinement of AA1050 Al alloy sheets by asymmetric rolling, Int. J. Mech. Sci. 50, 869-887 (2008)

[3] X. Liu, X.H. Liu, M. Song, X.K. Sun, L.Z. Liu, Theoretical analysis of minimum metal foil thickness achievable by asymmetric rolling with fixed identical roll diameters, Trans. Nonferrous Metals Soc. China 26, 501-507 (2016)

[4] I.F. Collins, P. Dewhurst, A slipline field analysis of asymmetrical hot rolling, Int. J. Mech. Sci. 17, 643-651 (1975) 
[5] M. Salimi, M. Kadkhodaei, Slab analysis of asymmetrical sheet rolling, J. Mater. Process. Technol. 150, 215-222 (2004)

[6] R. Shivpuri, P.C. Chou, C.W. Lau, Finite element investigation of curling in non-symmetric rolling of flat stock, Int. J. Mech. Sci. 30, 625-635 (1988)

[7] A.B. Richelsen, Numerical analysis of asymmetric rolling accounting for differences in friction, J. Mater. Process. Technol. 45, 149-154 (1994)

[8] Y.H. Ji, J.J. Park, W.J. Kim, Finite element analysis of severe deformation in $\mathrm{Mg}-3 \mathrm{Al}-1 \mathrm{Zn}$ sheets through differential-speed rolling with a high speed ratio, Mater. Sci. Eng. A 454-455, 570-574 (2007)

[9] C. Ma, L. Hou, J. Zhang, L. Zhang, Influence of thickness reduction per pass on strain, microstructures and mechanical properties of $7050 \mathrm{Al}$ alloy sheet processed by asymmetric rolling, Mater. Sci. Eng. A 650, 454-468 (2016)

[10] L. Ling, J. Tang, W. Liu, X. Zhang, L. Chen, Y. Deng, Numerical simulation evolution of shear strain and crystallographic textures during snake rolling of Al-Mg-Si alloy plate, J. Central South Univ. 48, 2281-2287 (2017)

[11] T. Zhang, Y. Wu, H. Gong, S.W. Ze, J.F. Min, Analysis of strain variation in cross shear zone of plate during snake hot rolling, J. Central South Univ. 24, 296-302 (2017)
[12] J. Yang, S. Li, J. Liu, X. Li, X. Zhang, Finite element analysis of bending behavior and strain heterogeneity in snake rolling of AA7050 plates using a hyperbolic sine-type constitutive law, J. Mater. Process. Technol. 240, 274-283 (2017)

[13] A. Aboutorabi, A. Assempour, H. Afrasiab, Analytical approach for calculating the sheet output curvature in asymmetrical rolling: In the case of roll axis displacement as a new asymmetry factor, Int. J. Mech. Sci. 105, 11-22 (2016)

[14] H.Y. Wang, Z.H. Wang, D.H. Zhang, D.W. Zhao, Analysis of snake rolling force and torque with changes of thickness depending on unequal roll radii based on pure aluminum experiments, Proc. Inst. Mech. Eng. 231, 161-174 (2017)

[15] X. Duan, T. Sheppard, Three dimensional thermal mechanical coupled simulation during hot rolling of aluminium alloy 3003, Int. J. Mech. Sci. 44, 2155-2172 (2002)

[16] M.R. Rokni, A. Zarei-Hanzaki, A.A. Roostaei, H.R. Abedi, An investigation into the hot deformation characteristics of 7075 aluminum alloy, Mater. Des. 32, 2339-2344 (2011)

[17] Q.Q. Zhang, Y.M. Liu, J. Yao, Numerical simulation of plate rolling process, Iron Steel Vanadium Titanium 25, 10-16 (2004)

[18] Y.W. He, Effect of the temperature field on deformation of aluminum alloys, Central South University, 2012

Cite this article as: P. Hao, J. Liu, Influence of snake rolling on metal flow in hot rolling of aluminum alloy thick plate, Mechanics \& Industry 21, 525 (2020) 